\title{
Blind SNR Estimation of Gaussian-Distributed Signals in Nakagami Fading Channels
}

\author{
Mohammed Hafez, Student Member, IEEE, Tamer Khattab, Member, IEEE, and \\ Hossam M. H. Shalaby, Senior Member, IEEE
}

\begin{abstract}
A blind (non-data-aided) SNR estimator using the statistical moments of the received signal is proposed. The proposed envelope-based non-data-aided estimator works for any time-domain Gaussian-distributed signal (e.g., OFDM signals). A closed-form expression for the estimated SNR as a function of the moments of the received signal and the Nakagami- $m$ parameter, is derived. Interestingly, the obtained expression shows that the proposed estimator operation and performance is independent of the constellation of the received signal. Moreover, the existence of the closed-form expression results in lower implementation complexity. Furthermore, to enable theoretical performance analysis, a general mathematical expression is derived for the even moments of the received signal in terms of SNR and the Nakagami- $m$ parameter. The performance of the proposed estimator is evaluated based on the mean-squared-error, under different conditions of the channel. An extension of our SNR estimation method into multiple antennas configurations is provided. Our results reveal that the proposed estimator works better in low SNR conditions, which is attractive to applications such as cognitive radio spectrum sharing scenarios.
\end{abstract}

Index Terms-Low SNR estimation, Gaussian-distributed signal, Nakagami fading, non-data-aided, time-variant channel.

\section{INTRODUCTION}

$\mathbf{K}$ NOWLEDGE of SNR at the receiver side is important due to its significance for several Maximum-Likelihood (ML) and Minimum-Mean-Square-Error (MMSE) techniques used in modern communication systems. The importance of knowing the instantaneous SNR increases with the use of adaptive techniques, power control, mobile-assisted hand-off, dynamic spectrum access, cognitive radio, and feedback-assisted resource allocation. The presence of the idea of cognitive radios introduces new techniques like spectrum sensing and sharing [1], [2]. Some of these methods use SNR as a mandatory parameter for their operation. Moreover, the nature of cognitive radios makes them work under low SNR, particularly in spec-

Manuscript received October 8, 2013; revised April 17, 2014 and August 25, 2014; accepted January 6, 2015. Date of publication February 12, 2015; date of current version July 8, 2015. The research work of Mohammed Hafez and Tamer Khattab has been funded by QNRF grant number NPRP 09-1168-2-455. The associate editor coordinating the review of this paper and approving it for publication was A. Wyglinski.

M. Hafez and T. Khattab are with the Department of Electrical Engineering, Qatar University, Doha, Qatar (e-mail: mohammed.hafez@qu.edu.qa; tkhattab@ieee.org).

H. M. H. Shalaby is with the Department of Electronics and Communications Engineering, Egypt-Japan University of Science and Technology (E-JUST), Alexandria 21934, Egypt, on leave from the Electrical Engineering Department, Alexandria University, Alexandria 21544, Egypt (e-mail: shalaby@ieee.org).

Color versions of one or more of the figures in this paper are available online at http://ieeexplore.ieee.org.

Digital Object Identifier 10.1109/TWC.2015.2403325 trum sharing scenarios, where a limit on cognitive user transmit power is imposed to control interference on primary users. A ML-SNR estimator for cognitive radios has been proposed in [4], which is used to control the transmitted power in a detect and avoid cognitive technique. Moreover, many studies have been done on the effect of SNR estimation on cognitive systems, with different system models and different realizations of the communication channel [3]-[6].

SNR estimation techniques can be categorized into DataAided (DA), Decision-Directed (DD), and Non Data-Aided (NDA) estimators. DA estimators like the ones presented in [7] use the transmitted pilot symbols to get their estimates. A DA estimator for Single Input Multi Output (SIMO) systems has been proposed in [9]. The existence of pilot symbols causes degradation in system throughput, which is the main disadvantage of this type of estimators. Moreover, in cognitive radio systems, where the primary users' pilot symbol locations might not be known to the secondary users, DA methods cannot be applied. DD estimators can be considered as a special case of DA estimators when the pilot symbols are replaced with the output of the decoder [7]. NDA estimators do not require knowledge of the transmitted signal, allowing in-service and inter-systems SNR estimation. An analytical expression for the exact Cramer-Rao lower bounds on the variance of unbiased NDA-SNR estimators of square QAM-modulated signals is given in [8].

SNR estimators can be categorized in another manner; namely, I/Q-based and envelope-based (EVB) estimators. I/Qbased estimators require coherent detection as they use inphase and quadrature components of the signal. I/Q estimators use the Expectation Maximization (EM) algorithm to get the ML estimate [10], [11]. The usage of the EM algorithm with SIMO systems has been introduced in [12]. The performance of I/Q-based estimators is greatly affected by I/Q imbalance. On the contrary, EVB estimators can be used with an I/Q imbalanced signal because they only make use of the signal magnitude. The resilience of EVB estimators to I/Q imbalance is a desired advantage in cases where SNR estimation is required before synchronization or equalization. EVB estimators exist for different channel models, different systems [7], [13][19], and using different higher order statistics [20], [21]. Also, extensions to SIMO schemes have been provided by [22]-[24].

For OFDM signals (structured signals), an estimator that makes use of some special properties (structure) of the cyclic prefix is presented in [25]. Such estimator cannot be considered blind since it requires a priori knowledge of the signal structure. Another estimator for OFDM signals that depends 
on frequency domain signal correlation is presented in [26]. Frequency-domain methods require knowledge of the signal constellation and suffer from larger delay as compared to timedomain methods.

SNR estimation for signals transmitted through Nakagami fading channels has been considered in [27]-[29]. A momentsbased EVB estimator in Nakagami- $m$ fading channels has been presented in [29]. The estimator has been found to malfunction under constant amplitude constellations (e.g., $M$-ary PSK signals), because the value of the fourth moment, of the constant amplitude constellations is equal to the square of the value of the second moment, rendering the ratio between the two moments a constant value, totally independent of the SNR. Moreover, the proposed SNR estimation technique presented in [29] depends on interpolation and lookup tables to find the SNR estimate, which implies higher mathematical complexity and more storage requirements. In addition, the estimator operation depends on the received signal constellation, which not only requires a priori knowledge of the received signal constellation, but also mandates an increase in the storage resources for lookup tables with the increase in the number of supported constellations, particularly for adaptive modulation systems.

In this paper, we utilize the same statistical moments-based EVB estimator presented in [29], but rather in the new context of SNR estimation for time-domain Gaussian-distributed signals (OFDM signal is a subset of this signal category):

- The newly proposed SNR estimation context utilizes the received time domain signal to get an estimate of the SNR, which eliminates the need for synchronization.

- When used for Gaussian-distributed signals (e.g., OFDM signals), the operation and performance of the momentsbased estimator are shown to be independent of the received signal constellation.

- This enables its operation under all types of signal constellations including constant amplitude constellations (e.g., $M$-ary PSK), in contrast to the results in [29].

- We are able to derive a general formula that represents the SNR estimate as a function of the statistical moments of the received time-domain signal, which is directly related to the Nakagami- $m$ parameter.

- The derived mathematical formula provides a proof that the estimator operation is independent of signal constellations.

- Also, it enables an analytical performance evaluation of the SNR estimation technique.

- And, it provides a very low complexity implementation alternative to the resource expensive lookup table and the computationally expensive interpolation operations, which are mandatory in the system presented in [29].

- Furthermore, in our analysis, we take into account the effects of the size of the data used to estimate the received signal statistics on the estimator's performance, the multipath environment, the Doppler spread, and the mismatch in the estimation of the $m$ parameter of the Nakagami- $m$ channel gain distribution.

- For the sake of completeness of our work, we extend our context to different multiple antennas configurations; Single-Input-Multiple-Output (SIMO), Multiple-InputSingle-Output (MISO), and Multiple-Input-MultipleOutput (MIMO).

The rest of the paper is organized as follows: Section II describes the system model. Section III carries the derivation for the estimation formula of the new estimator context and its extension to the multiple antennas schemes. Section IV considers the effects of the multi-path channel and Doppler spread. In Section V, simulation results are presented. Finally, the conclusion is given in Section VI.

\section{SyStem Model}

We consider a digital communication system over a timevariant (fast fading) channel, where the coherence time of the channel, $\tau_{c}=1 / f_{d}$, where $f_{d}$ is the Doppler spread, is smaller than the transmitted symbol duration (in case of OFDM transmission this will be the OFDM symbol length). This is a typical channel for high speed mobility scenarios or slow rate systems [30]. In addition, we assume that the channel delay spread, $\tau_{d}$, is much smaller than the symbol duration. This implies that inter-symbol-interference, ISI, is ignored (which is the case for $\tau_{d}$ smaller than the length of the cyclic-prefix for OFDM signals). The received signal $r_{n}$ is given by

$$
r_{n}=g_{n} s_{n}+w_{n}, \quad n=0, \ldots, N-1,
$$

where $N$ is the number of samples in a symbol, referred to as the symbol size ( $F F T$ size in case of OFDM), $g_{n}$ is the fading channel gain, $s_{n}$ is the transmitted signal samples and $w_{n}$ is a complex zero-mean white Gaussian noise with a variance equal to $2 \sigma_{w}^{2}$.

The channel gain, $g_{n}$, is modeled as a zero-mean complex random variable and can be written as $g_{n}=\left|g_{n}\right| e^{j \phi}$ with $\phi$ taking values between $-\pi$ and $\pi$ under any arbitrary distribution. We are not concerned about the distribution of the phase, as we are using an EVB estimator, which will not be affected by the phase of the signal. $\left|g_{n}\right|$ follows the Nakagami- $m$ distribution:

$$
f_{\left|g_{n}\right|}\left(\left|g_{n}\right|\right)=\frac{2}{\Gamma(m)}\left(\frac{m}{\alpha_{g}^{2}}\right)^{m}\left|g_{n}\right|^{2 m-1} \exp \left(-\frac{m\left|g_{n}\right|^{2}}{\alpha_{g}^{2}}\right),
$$

where $\alpha_{g}^{2}=E\left(\left|g_{n}\right|^{2}\right)$ and $\Gamma($.$) is the Gamma function. It$ should be noticed that $\left|g_{n}\right|$ and $\phi$ are independent. The Nakagami- $m$ distribution approximates a Rician distribution for the fading parameter $m$ in the range $1<m<\infty$, and approximates a Hoyt or Nakagami- $q$ distribution for $m$ in the range $0.5 \leq m<1$, while it becomes a Rayleigh distribution for $m=1$ [31]. This makes the Nakagami- $m$ distribution a more generalized case, that includes other commonly used Rayleigh and Rician fading channels.

Generally, moment based estimators are suitable for Gaussiandistributed signals (OFDM signals are Gaussian-distributed 
signals as shown in Appendix B). A Gaussian-distributed signal

$$
s_{n}=I_{n}+j Q_{n}
$$

is a signal where the real $I_{n}$ and imaginary $Q_{n}$ parts of the complex time domain signal are both Gaussian random variables. For this type of signals, the envelope $\left|s_{n}\right|$ follows the Rayleigh distribution,

$$
f_{\left|s_{n}\right|}\left(\left|s_{n}\right|\right)=\frac{\left|s_{n}\right|}{\sigma^{2}} e^{-\frac{\left|s_{n}\right|^{2}}{2 \sigma^{2}}},
$$

where $\sigma^{2}=\operatorname{Var}\left(I_{k}\right)=\operatorname{Var}\left(Q_{k}\right)=\frac{1}{2}$, for a unit power transmitted signal. Hence, the SNR can be expressed as:

$$
\rho=\frac{\alpha_{g}^{2}}{2 \sigma_{w}^{2}} .
$$

\section{Proposed Estimator}

The idea is to get an estimate of the SNR using the ratio between the different moments of the received time-domain signal, which is widely known as the method of moments [32]. The probability density function of $r_{n}$, conditioned on both $g_{n}$ and $s_{n}$, is given by [33]

$$
\begin{array}{r}
p\left(\mid r_{n} \| g_{n}, s_{n}\right)=\frac{\left|r_{n}\right|}{\sigma_{w}^{2}} \exp \left(-\frac{\left|g_{n}\right|^{2}\left|s_{n}\right|^{2}+\left|r_{n}\right|^{2}}{2 \sigma_{w}^{2}}\right) \\
\quad \times I_{0}\left(\frac{\left|r_{n}\right|\left|g_{n}\right|\left|s_{n}\right|}{\sigma_{w}^{2}}\right),
\end{array}
$$

where $I_{0}($.$) is the modified Bessel function of the first kind and$ order zero. This leads to

$$
\begin{aligned}
E\left[\left|r_{n}\right|^{l}\right]= & \int_{0}^{\infty} 2^{\frac{l}{2}} \Gamma\left(\frac{l}{2}+1\right) \sigma_{w}^{l} e^{-\left|s_{n}\right|^{2}}\left(\frac{m}{m+\rho\left|s_{n}\right|^{2}}\right)^{m} \\
& \times{ }_{2} F_{1}\left(\frac{l}{2}+1, m, 1 ; \frac{\rho\left|s_{n}\right|^{2}}{m+\rho\left|s_{n}\right|^{2}}\right) d\left|s_{n}\right|^{2},
\end{aligned}
$$

where ${ }_{2} F_{1}(a, b, c ; x)$ is the Gauss hypergeometric function [34], (Refer to Appendix A for the steps leading to (7)).

As shown in Fig. 1, we use the ratio between the fourth and the second moments of the time domain signal to get the estimate of the SNR. Defining $z$ as the estimation parameter, we get

$$
\begin{aligned}
z & =\frac{M_{4}}{M_{2}^{2}}=f(\rho), \\
M_{l} & =E\left[\left|r_{n}\right|^{l}\right], \quad l \in\{2,4\} \\
\text { and } \quad \rho & =f^{-1}(z) .
\end{aligned}
$$

These moments can be calculated using the following theorem:

Theorem 1: The even moments of the received Nakagami- $m$ faded OFDM signal can be produced using

$$
M_{2 x}=2^{x} \Gamma(x+1) \sigma_{w}^{2 x}\left(1+\sum_{j=1}^{x}\left(\begin{array}{c}
x \\
j
\end{array}\right) \frac{m^{(j)}}{m^{j}} \rho^{j}\right),
$$

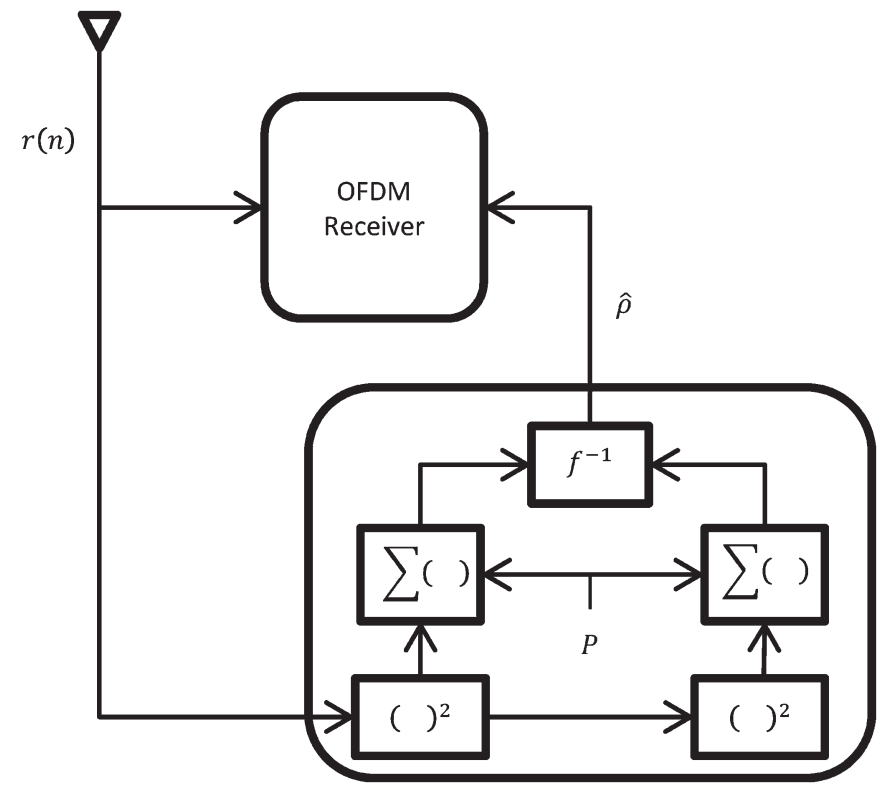

Fig. 1. Functional block diagram of the proposed estimator. Each block is annotated by the operation it performs on its input to produce its output.

where $m^{(j)}$ is Pochhammer's operator, and $m \in \mathbb{R}$.

Proof: The proof is conducted in Appendix A.

As $M_{l}$ is not available in practice, we can use the following estimate based on the symbols of the received signal

$$
\hat{M}_{l}=\frac{1}{P} \sum_{p=1}^{P}\left|r_{p}\right|^{l}, \quad l \in\{2,4\},
$$

where $P$ is the number of data symbols used to get the estimate of the moments. Hence,

$$
\begin{aligned}
\hat{z} & =\frac{\hat{M}_{4}}{\hat{M}_{2}{ }^{2}} \\
\text { and } \quad \hat{\rho} & =f^{-1}(\hat{z}) .
\end{aligned}
$$

The inverse function can be implemented using lookup tables, polynomial approximation or direct formula. The independence of this estimator of the signal constellation gives it the advantage of being usable in adaptive systems, as only one inverse function is needed for all different constellations. We choose $l \in\{2,4\}$, because using higher order moments will produce a more complex relation between $\rho$ and $z$, which can be noninvertible. Also it does not provide significant improvement in the performance of the estimator to justify the added complexity by the higher order moments. Moreover, even if the higher moments provide some performance gain in the linear region, it may make the linear region of the relation span a shorter range of the SNR. Fig. 1 shows that the estimator is simple, easy to implement, needs small memory resources, and its complexity is linearly related to the number of data symbols used for estimation (i.e., the complexity of the estimator is in the order of $P$ ). The estimator can be easily implemented using the algorithm in Table I. 
TABLE I

ESTIMATOR BLOCK IMPLEMENTATION ALgORITHM

\begin{tabular}{|c|c|}
\hline initiate $P$ & $\begin{array}{l}\text { based on the acceptable error margin as } \\
\text { explained in comments on Figure } 6 \text { in } \\
\text { Section } V \text {. }\end{array}$ \\
\hline \multicolumn{2}{|r|}{ 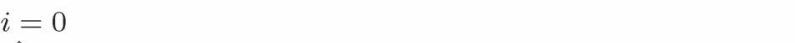 } \\
\hline \multicolumn{2}{|l|}{$\hat{M}_{2}=0$} \\
\hline \multirow{2}{*}{\multicolumn{2}{|c|}{$\begin{array}{l}\hat{M}_{4}=0 \\
\text { while } i<P\end{array}$}} \\
\hline & \\
\hline \multicolumn{2}{|c|}{$x=a b s\left(r_{i}\right)^{2}$} \\
\hline \multicolumn{2}{|c|}{$y=x^{2}$} \\
\hline \multicolumn{2}{|c|}{$\hat{M}_{2}=\hat{M}_{2}+x$} \\
\hline \multicolumn{2}{|c|}{$\begin{array}{l}\hat{M}_{4}=\hat{M}_{4}+y \\
i=i+1\end{array}$} \\
\hline \multicolumn{2}{|l|}{ end } \\
\hline \multicolumn{2}{|l|}{$\hat{z}=\frac{\hat{M}_{4}}{\hat{M}^{2}}$} \\
\hline$\hat{\rho}=f^{-1}(\hat{z})$ & achived by plugging $\hat{z}$ in place of $z$ in (15) \\
\hline
\end{tabular}

Based on Theorem 1, we can rewrite (8) and (10) as

$$
z=2 \frac{\left(1+2 \rho+\gamma_{m} \rho^{2}\right)}{\left(1+2 \rho+\rho^{2}\right)}
$$

and

$$
\rho=\frac{z-2}{2 \gamma_{m}-z}+\frac{\sqrt{2} \sqrt{(z-2)\left(\gamma_{m}-1\right)}}{2 \gamma_{m}-z}, \quad 2<z<2 \gamma_{m},
$$

where $2 \gamma_{m}$ is the upper limit of the estimation parameter, which is related to the Nakagami- $m$ parameter through the following relation

$$
\gamma_{m}=\frac{m+1}{m}
$$

Here, we assume perfect knowledge of the value of $m$, which can be estimated using one of the methods presented in [35]. Although, the methods in [35] are considering Nakagami distributed observations, which is not the case for our model, modifications for these methods to suite our model, and joint estimation of the Nakagami- $m$ parameter and the SNR, can be considered for future work. Beside that, Theorem 1 shows that this estimator is totally independent of the signal constellation; we can see that the moments of the received signal are only related to the average SNR and the Nakagami- $m$ shaping parameter. It can be easily shown that (15) is also valid for the case where the system is affected by a Gaussian interference. In this case $\rho=\frac{\alpha_{g}^{2}}{2\left(\sigma_{w}^{2}+\sigma_{I}^{2}\right)}$, where $\rho$ represents the Signal to Interference plus Noise Ratio (SINR) and $\sigma_{I}^{2}$ is the power of Gaussian interference.

\section{A. Multiple Antennas Schemes}

Here, we introduce the modifications needed for our estimator to work under the usage of multiple-antennas configurations. We also show in the results section that the estimator performance gains from the diversity presented by these schemes.

1) SIMO: Consider a system with $N_{R}$ receiving antennas and all branches have the same average noise power $\sigma_{w \_S I M O}^{2}$. Let $\hat{\sigma}_{w_{i}}^{2}$ be the estimated average noise power of the $i^{t h}$ branch, $i=1,2, \ldots, N_{R}$. The SNR on the $i^{t h}$ receive branch is given by

$$
\rho_{i}=f^{-1}\left(z_{i}\right)=f^{-1}\left(\frac{M_{4_{i}}}{M_{2_{i}}^{2}}\right)
$$

where $M_{l_{i}}$ is the $l^{t h}$ moment of the signal received on the $i^{t h}$ branch. Using Theorem 1, we can find that

$$
\hat{\sigma}_{w_{i}}^{2}=\frac{\hat{M}_{2_{i}}}{2\left(1+\hat{\rho}_{i}\right)}
$$

and taking the average of the estimated noise power over all the $N_{R}$ antennas results in a better estimate of the average noise power over the branches, which will enhance the estimated $\mathrm{SNR}$. The average noise power is given by

$$
\hat{\sigma}_{w \_S I M O}^{2}=\frac{1}{N_{R}} \sum_{i=1}^{N_{R}} \hat{\sigma}_{w_{i}}^{2}
$$

and the enhanced estimated SNR of the $i^{t h}$ branch becomes

$$
\hat{\rho}_{i \_S I M O}=\frac{\hat{\rho}_{i} \times \hat{\sigma}_{w_{i}}^{2}}{\hat{\sigma}_{w \_S I M O}^{2}} .
$$

2) MISO: Here, we have a transmitter that has $N_{T}$ transmitting antennas and uses repetition coding. We assume that the $N_{T}$ channels between the transmitter and the receiver are independently-identically-distributed (i.i.d.) (i.e., all the channels have the same value of $m$, and independent from each other). Hence, the received signal can be written as

$$
r_{n}=s_{n} \sum_{i=1}^{N_{T}} g_{n}^{(i)}+w_{n}
$$

where $g_{n}^{(i)}$ is the channel gain at the $i^{\text {th }}$ transmit antenna. Because of this configuration, the average power of the signal transmitted on the $i^{t h}$ transmit antenna is $\sigma_{s_{i}}^{2}=\frac{1}{2 N_{T}}$ and $\alpha_{g}^{2}=$ $\sum_{i=1}^{N_{T}} \alpha_{g_{i}}^{2}$, where $\alpha_{g_{i}}^{2}$ is the average power gain of the channel experienced by the signal transmitted from the $i^{t h}$ antenna. Then

$$
\rho=\frac{\alpha_{g}^{2} \sigma_{s}^{2}}{\sigma_{w}^{2}} .
$$

Reconsidering (4), (6), (7), and (13) under this configuration, we get the same expression for $\rho$, as the one in (15), but with

$$
\gamma_{m}=\frac{\left(2 N_{T}-1\right) m+1}{N_{T} m} .
$$

3) MIMO: Now, we will discuss two schemes for MIMO configuration, namely, transmit diversity and spatial multiplexing.

Starting with the transmit diversity scheme, we can find that it is a direct cascading for the two previous configurations (i.e., a MISO system followed by a SIMO system). In this case, the estimator will benefit from the diversity presented by the MISO system to get an enhanced performance especially at the low mobility case, and the existence of the SIMO system will provide us with a more accurate estimate for the noise power at the receiver.

The spatial multiplexing scheme also can be considered as a combination of the MISO and SIMO configurations. This combination is formed by $J$ different MISO systems parallel to each others, where $J$ is the number of transmitted streams, 
and all of them are cascaded with a single SIMO system. Each MISO system contains $N_{T_{j}}$ transmitting antennas dedicated for the $j^{\text {th }}$ stream and the SIMO system has $N_{R}$ receiving antennas, where $N_{R} \geq J$. Then, the estimated SINR at the $i^{t h}$ receiving antenna is

$$
\rho_{i, j}=\frac{{ }^{(i)} \alpha_{g_{j}}^{2}}{2\left(\sigma_{w_{i}}^{2}+\sigma_{I_{j, i}}^{2}\right)},
$$

where ${ }^{(i)} \alpha_{g_{j}}^{2}=\sum_{k=1}^{N_{T_{j}}(i)} \alpha_{g_{j, k}}^{2}$ is the average channel gain experienced by the $j^{t h}$ transmitted stream, and $\sigma_{I_{j, i}}^{2}=$ $\sum_{n=1, n \neq j}^{J}{ }^{(i)} \alpha_{g_{n}}^{2}$ is the interference caused by the transmission of the other streams on the $j^{\text {th }}$ stream.

Again using Theorem 1, we find that

$$
{ }^{(i)} \alpha_{g_{j}}^{2}=\frac{\rho_{i, j} M_{2_{i}}}{1+\rho_{i, j}},
$$

assuming that all channels are i.i.d.. Hence, the estimated average channel gain is

$$
\hat{\alpha}_{g_{j} \_ \text {MIMO }}^{2}=\frac{1}{N_{R}} \sum_{i=1}^{N_{R}}{ }^{(i)} \hat{\alpha}_{g_{j}}^{2},
$$

and

$$
\hat{\rho}_{i, j \_M I M O}=\frac{\hat{\rho}_{i, j} \times \hat{\alpha}_{g_{j \_} M I M O}^{2}}{{ }^{(i)} \hat{\alpha}_{g_{j}}^{2}},
$$

where the superscript ${ }^{(i)}[$.$] indicates that the process is related$ to the $i^{t h}$ receiving antenna. Using the same set of equations, we can get separate estimates for $\sigma_{w}^{2}$ and $\sigma_{I_{j}}^{2}$.

\section{EfFects of Multi-Path and Doppler Spread}

Modifying the system model to consider multi-path effect leads to

$$
r_{n}=\sum_{i=0}^{L-1} g_{n-\tau_{i}}^{i} s_{n}+w_{n}, \quad n=0, \ldots, N-1,
$$

where $L$ is the number of the independent paths of the channel, $g_{n-\tau_{i}}^{i}$ is the channel gain of the $i^{t h}$ path at the time $\left(n-\tau_{i}\right)$ and $\tau_{i}$ is the delay of the $i^{t h}$ path. This can be considered the same as the MISO system case, assuming that all paths have the same average power, which is the worst case scenario, and the maximum delay is within the cyclic-prefix length. The increase in the number of received paths (the number of transmitting antennas for the MISO system) would add more variations to the received signal, which can be considered as an advantage for our estimator. These variations enhance the estimator's performance, especially in the case of slow fading channels.

Now, we need to demonstrate the effect of the Doppler spread on the system. As the estimator is not designed for a certain system with a certain rate, we can express the channel coherence time in terms of the system data rate

$$
T_{c}=q T_{s}, \quad q>0,
$$

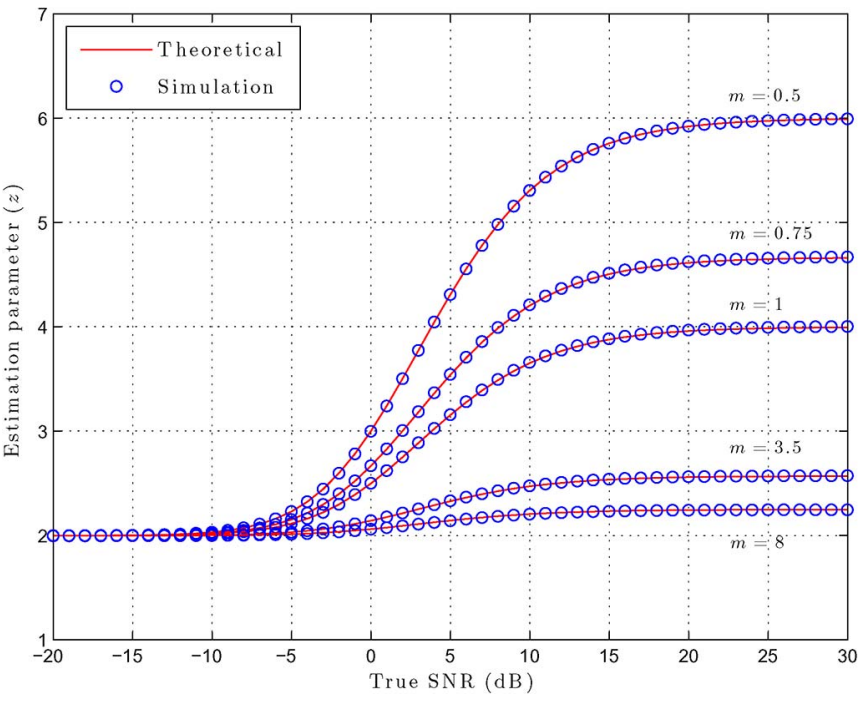

Fig. 2. Relation between the estimation parameter $z$ and SNR.

where $T_{c}$ is the channel coherence time, $T_{s}$ is the data symbol time and $q$ is an integer value. In the previous analysis, we considered a time-variant channel with a very short coherence time (i.e., $q=1$ ). With the increase of the value of $q$, the channel becomes more static over the data samples. A channel with a very long coherence time (i.e., $q \rightarrow \infty$ ) has the same effect as a Nakagami fading channel with $m \rightarrow \infty$. This is the situation where the estimation parameter is constant over the whole range of the SNR and the estimator would fail. Therefore, increasing the channel coherence time (i.e., smaller Doppler spread) lowers the estimator's performance.

\section{Numerical Results}

In Fig. 2, we draw the relation between the estimation parameter $z$ (as defined in (8)) and the SNR from simulation using (12) and from theoretical solution using (14) and (16). This is done for a set of different values of the Nakagami- $m$ parameter, and the results are almost identical.

Fig. 3 shows the change of the estimator performance in terms of the Normalized-Root-Mean-Squared-Error NRMSE with different values of the Nakagami- $m$ parameter.

$$
N R M S E=\frac{\sqrt{E\left[(\hat{\rho}-\rho)^{2}\right]}}{\rho} .
$$

The performance is better in the severe fading cases (i.e., low values of $m$ ) and in the low SNR range, which renders this estimator suitable for cognitive radios, because of their transmit power constraints. This result is consistent with the results in Fig. 2, where the range of values that the estimation parameter can take decreases with the increase of $m$. The smaller range increases the probability of error, causing performance degradation. Also, it is clear from (14) and (16) that increasing the value of $m$ results in $\gamma_{m} \rightarrow 1$, which makes the estimation parameter $z$ less representative of the SNR. 


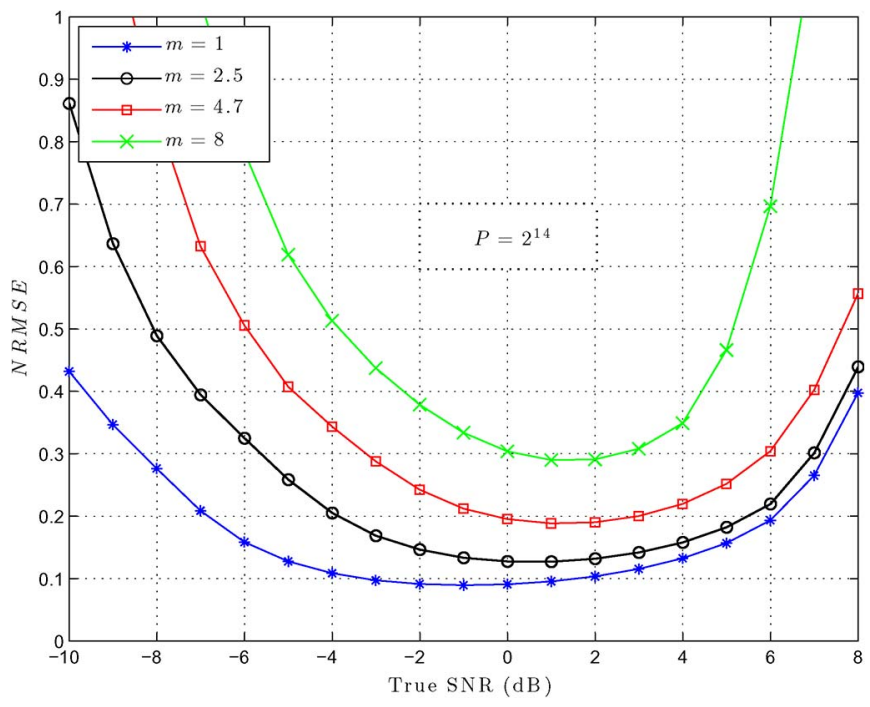

Fig. 3. NRMSE of the estimator for different values of $m$.

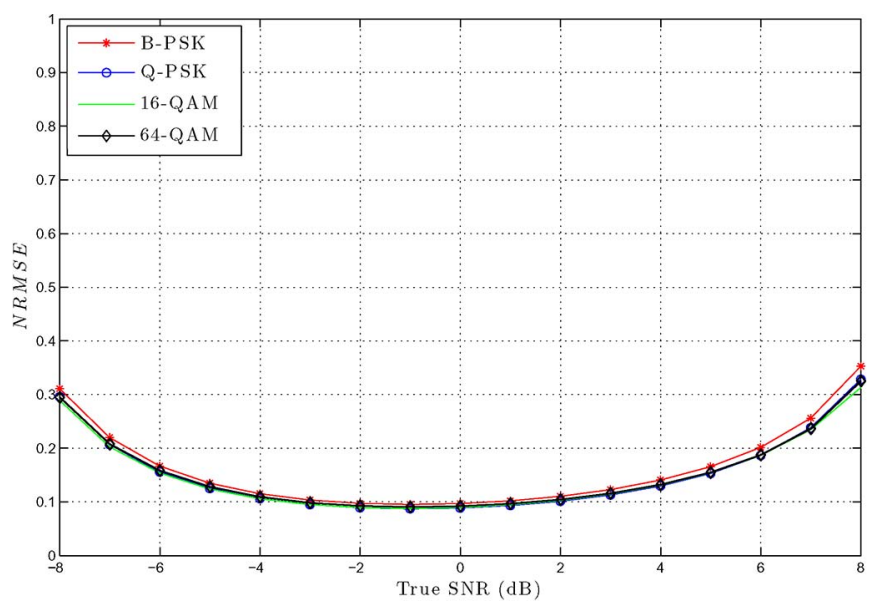

Fig. 4. NRMSE of the estimator for different constellations of the transmitted signal.

Fig. 4 presents a comparison for the performance of the estimator with different constellations for the transmitted signal. We can see that the performance is not affected by the change in the signal constellation. As evident from Theorem 1, the only parameter related to the transmitted signal, hence affecting the relation between the moments and the SNR, is the average signal power while different levels taken by the constellation do not affect the relation.

Fig. 5 shows the increase in the estimated SNR error, which is produced by the mismatch in the estimated Nakagami-m parameter. The mismatch is measured as the percentage of error in the value of $m$. We can see that when we hit $50 \%$ difference in the value of the $m$-parameter, the error in the estimated SNR increases about $50 \%$. This shows that we have small tolerance value around small values of $m$, and this tolerance increases with the increase of the value of $m$.

Fig. 6 demonstrates the effect of the size of the data used for the estimation of the received signal statistics. Increasing the size enhances the performance over the whole range of SNR values. It is clear that the estimator would reach zero error as $P \rightarrow \infty$, which is not practical. The amount

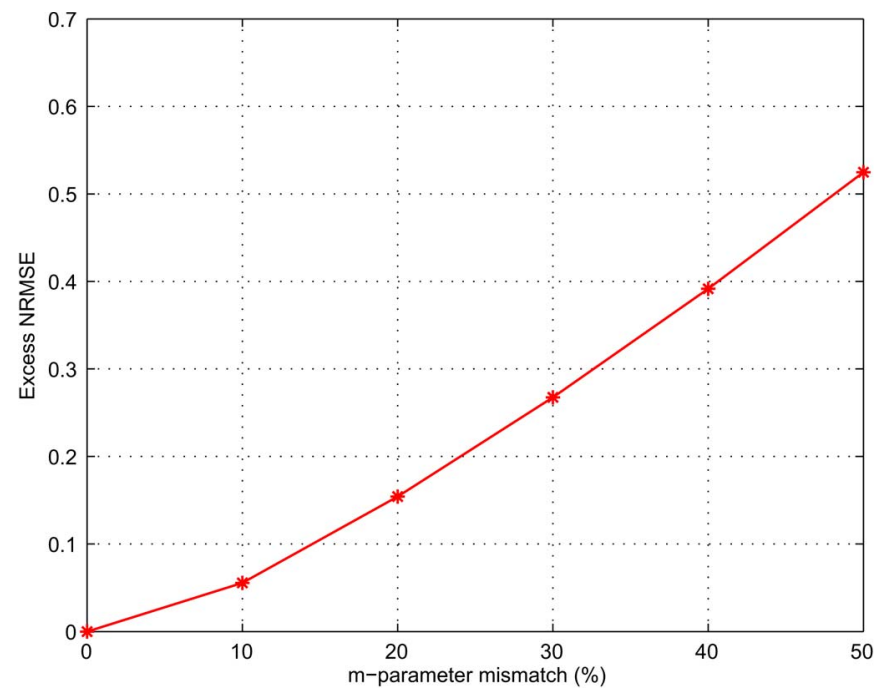

Fig. 5. The effect of mismatch in the Nakagami $m$-parameter.

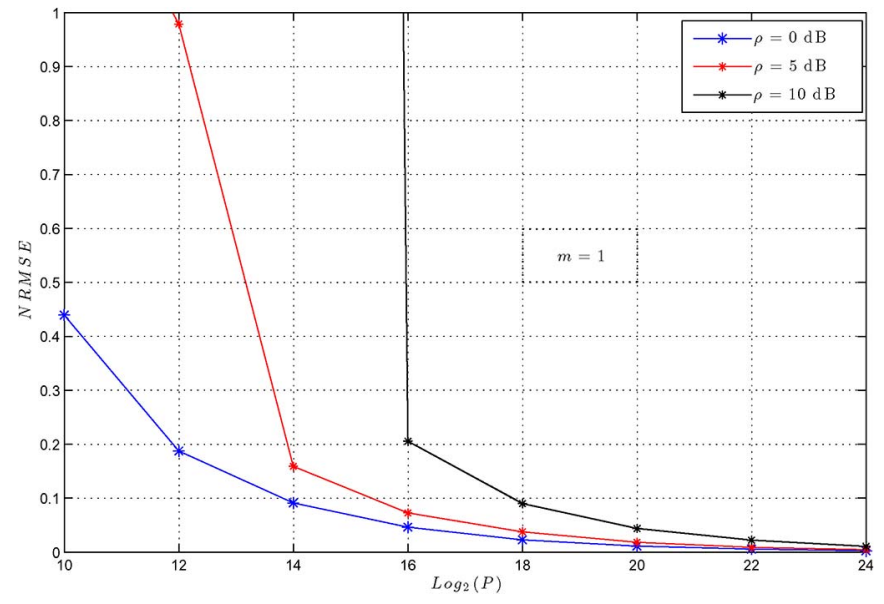

Fig. 6. Effect of the size of data used for estimation.

of data symbols needed to perform the estimation can be chosen based on the application and its sensitivity to errors. As an example, if we consider the effect of the SNR mismatch on turbo decoding, we can find that an error within $1 \mathrm{~dB}$ at low SNR range (below $5 \mathrm{~dB}$ ) has a very small effect on the performance of the decoder [13]. This error limit represents a maximum value of 0.4 of the NRMSE in the case of our estimator, which is obtained by taking $P=2^{12}$ (i.e., for a system working at a rate of $50 \mathrm{Mbps}$, it needs less than $0.5 \mathrm{~ms}$ to get a good estimate of the SNR).

From Fig. 2 and Fig. 6, it is obvious that the best performance of the estimator is around $0 \mathrm{~dB}$, which is the region where the relation between the estimation parameter and the SNR takes a linear shape. The non-linearity of the relation in the upper and lower ranges of the SNR causes the estimator to be biased and to lose performance. This can be explained from (14), where for very low values of $\rho$, the terms containing $\rho$ in (14) can be neglected, therefore $z=2$. In the same manner, very high values of $\rho$ makes the second order term the dominant term, which leads to $z=2 \gamma_{m}$. As a result, the performance of the estimator in upper and lower ranges of the SNR degrades due to the saturation of the estimation parameter value. According 


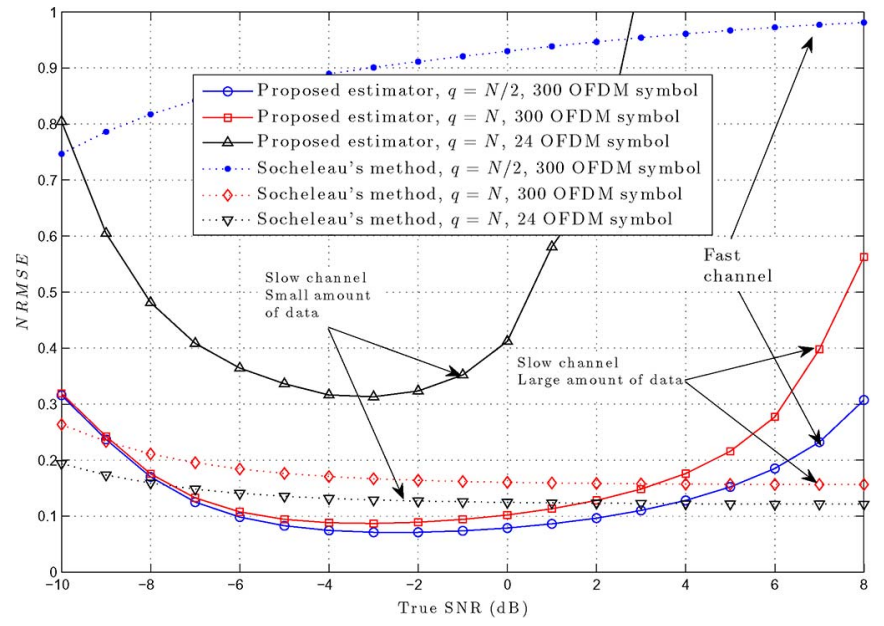

Fig. 7. Performance comparison between the proposed estimatorand the other estimator in [25].

to Fig. 6, this range can be extended by increasing the amount of data used for estimation to provide more accurate estimates of the moments, which overcomes the problem of the non-linear part of the relation. This can be further clarified by investigating the nature of the error. Here, $\hat{z}$ can be considered as the kurtosis of the received signal; then we can think of it as a Gaussian random variable with the mean equal to the true value of $z$ and the variance inversely proportional to the number of the samples. Thus, in the case of small number of samples, the variance of $\hat{z}$ is large and its value fluctuates in a wide range, which will lead to a big variation in the value of $\hat{\rho}$, especially in the non-linear region. This again reflects on Fig. 6 and can be seen by the non smooth performance for the high SNR values.

Fig. 7 compares the SNR performance of the estimator described in [25] with our proposed estimator. This simulation is done using $N=1024, L=3$, and $m=1$. The method proposed in [25], which shows the best performance in the literature for OFDM signals, depends on the periodic redundancy induced by the cyclic-prefix. This makes it sensitive to any major change in the channel within the OFDM symbol. A channel with short coherence time will cause the cyclic-prefix samples and the corresponding data samples to be uncorrelated, hence diminishing the redundancy induced by the cyclic-prefix. Socheleau's method has an advantage when the channels have small Doppler spread. Our estimator has a better performance in large Doppler spread cases, and it has a competitive performance in the smaller Doppler spread cases if the number of data samples $P$ is large enough. Beside that, our estimator has the advantage of working without need for a previous synchronization process.

Fig. 8 shows the NRMSE for the estimated SNR on one of the antenna branches in the SIMO system. It clearly shows that the SIMO system takes advantage of the diversity to gain a more accurate estimate of the noise power, resulting in more accurate estimate of the SNR. The estimate becomes more accurate by increasing the number of receiving antennas.

Fig. 9 presents the performance of our estimator in the case of a MISO system. It shows that we do not get better performance for the cases where $m$ has a small value. Actually, we may lose

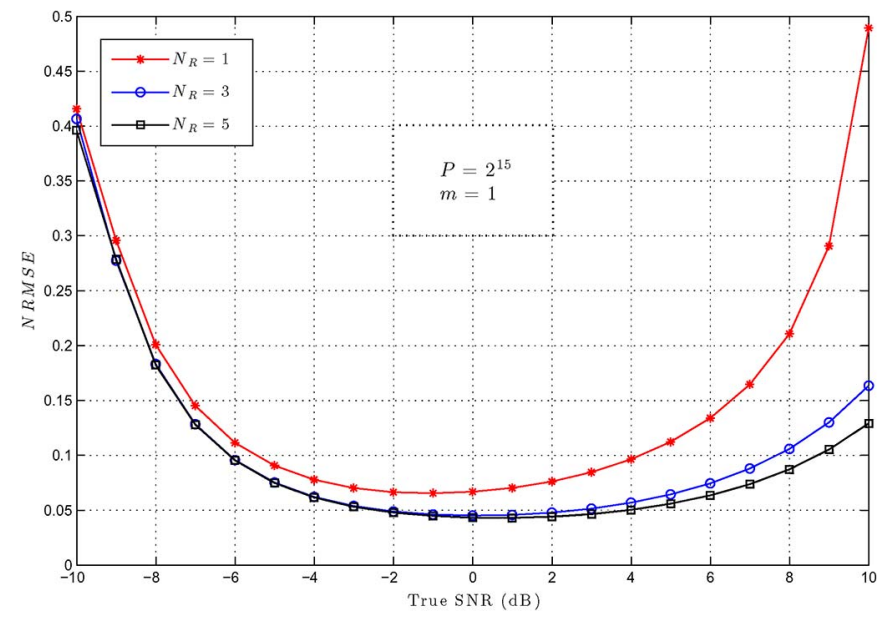

Fig. 8. The performance of the estimator under the SIMO configuration.

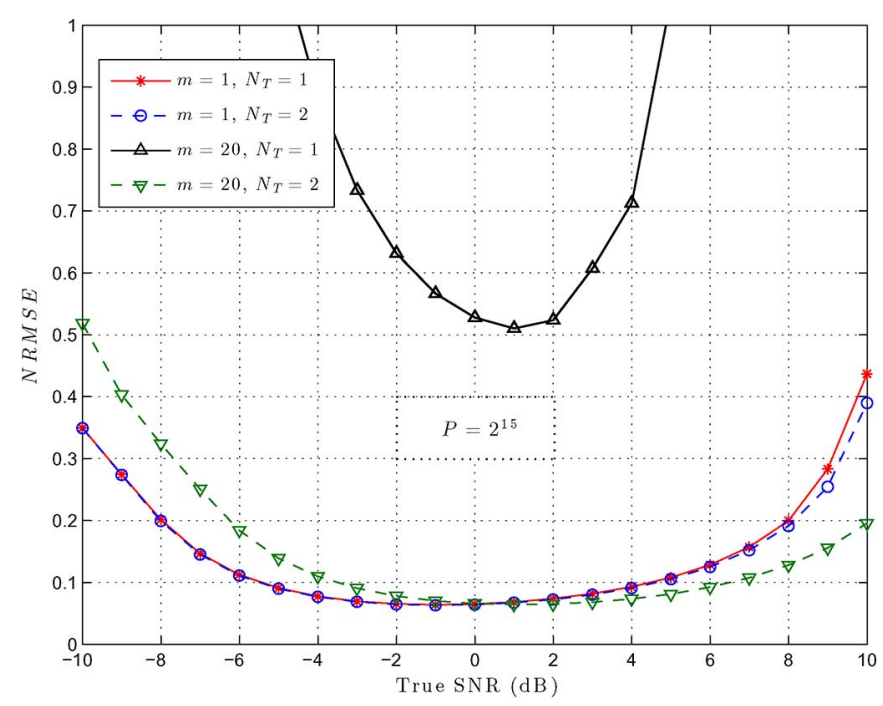

Fig. 9. The performance of the estimator under the MISO configuration.

some performance for very small $\mathrm{m}$. Contrary, for the large values of $m$, there is a big jump in the performance just by using one additional transmitter. As mentioned in Section IV, this can be applied also for the Multi-path channel. In the multi-path channel, our estimator will maintain good performance, even in low mobility (large coherence time) cases.

Fig. 10 includes the performance of the estimator for all antenna configurations; namely SIMO, MISO, and transmit diversity MIMO.

\section{CONCLUSION}

A blind and constellation-independent SNR estimator that works in time-domain for Gaussian-distributed signals has been proposed. Generalized formulas for the estimator and the moments of the received signal, that only depends on the Nakagami- $m$ parameter, has been given. Our results reveal that this estimator is suitable for cognitive radios, as it works better in low SNR scenarios, especially in time-varying channels. The performance mainly depends on the size of the data used to estimate the received signal statistics; yet, it has been shown that a good performance can be achieved in a reasonable time. 


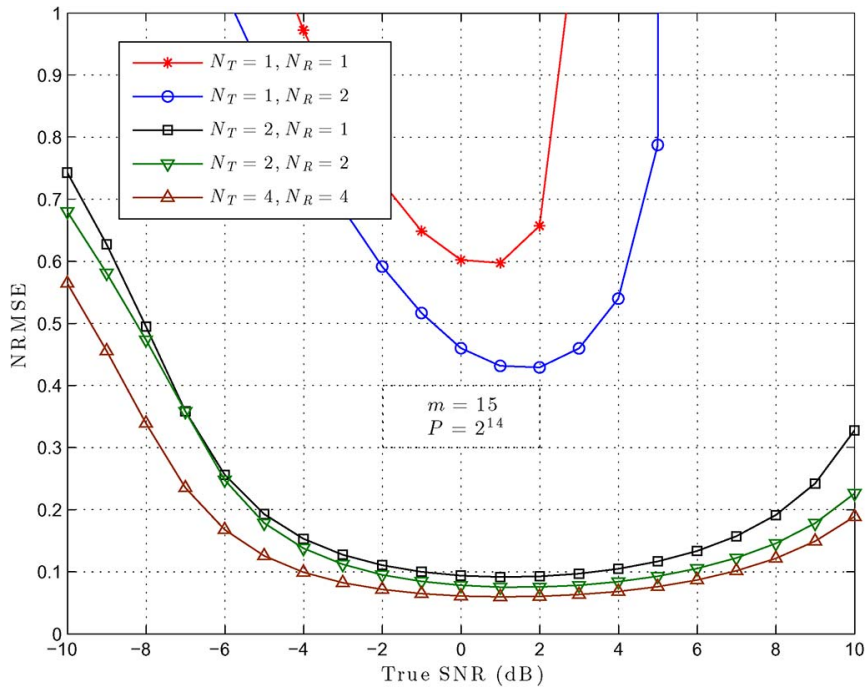

Fig. 10. The performance of the estimator for different antenna configurations.

Also, the estimator can gain benefit from the diversity presented in the systems with multiple-antennas and can be used in Gaussian interference channels. The effect of mismatch in the estimation of the Nakagami- $m$ parameter has been examined, as well, and the results show that it has a slight effect on the performance.

\section{APPENDIX A}

\section{PROOF OF THEOREM 1}

Starting from (6), the conditional expected value for the $l^{t h}$ moment of the received signal is

$$
\begin{aligned}
E\left[\left|r_{n}\right|^{l} \mid g_{n}, s_{n}\right]= & \int_{0}^{\infty}\left|r_{n}\right|^{l} p\left(\left|r_{n}\right| \mid g_{n}, s_{n}\right) d\left|r_{n}\right| \\
= & e^{-\frac{\left|g_{n}\right|^{2}\left|s_{n}\right|^{2}}{2 \sigma_{w}^{2}}} \int_{0}^{\infty} \frac{\left|r_{n}\right|^{l+1}}{\sigma_{w}^{2}} e^{-\frac{\left|r_{n}\right|^{2}}{2 \sigma_{w}^{2}}} \\
& \times I_{0}\left(\frac{\left|g_{n}\right|\left|s_{n}\right|}{\sigma_{w}} \times \frac{\left|r_{n}\right|}{\sigma_{w}}\right) d\left|r_{n}\right| \\
= & e^{-\frac{\left|g_{n}\right|^{2}\left|s_{n}\right|^{2}}{2 \sigma_{w}^{2}} \sigma_{w}^{l} \int_{0}^{\infty} x^{l+1} e^{-\frac{x^{2}}{2}}} \\
& \times I_{0}\left(\frac{\left|g_{n}\right|\left|s_{n}\right|}{\sigma_{w}} \times x\right) d x,
\end{aligned}
$$

where $\frac{\left|r_{n}\right|}{\sigma_{w}}=x$. Based on [36, section 6.631]

$$
\begin{aligned}
\int_{0}^{\infty} x^{\mu} e^{-\alpha x^{2}} J_{v}(\beta x) d x= & \frac{\beta^{v} \Gamma\left(\frac{v}{2}+\frac{\mu}{2}+\frac{1}{2}\right)}{2^{v+1} \alpha^{\frac{1}{2}(\mu+v+1)} \Gamma(v+1)} \\
& \quad \times{ }_{1} F_{1}\left(\frac{v+\mu+1}{2} ; v+1 ;-\frac{\beta^{2}}{4 \alpha}\right)
\end{aligned}
$$

and $I_{0}(x)=J_{0}(j x)$.
Hence,

$$
\begin{aligned}
E\left[\left|r_{n}\right|^{l} \mid g_{n}, s_{n}\right]=e^{-\frac{\left|g_{n}\right|^{2}\left|s_{n}\right|^{2}}{2 \sigma_{w}^{2}}} \sigma_{w}^{l} 2^{\frac{l}{2}} \Gamma\left(\frac{l}{2}+1\right) \\
\\
\quad \times{ }_{1} F_{1}\left(\frac{l}{2}+1 ; 1 ; \frac{\left|g_{n}\right|^{2}\left|s_{n}\right|^{2}}{2 \sigma_{w}^{2}}\right) .
\end{aligned}
$$

To find the unconditional moment, we start by removing the condition on $g_{n}$,

$$
\begin{aligned}
E\left[\left|r_{n}\right|^{l} \mid s_{n}\right] & =\int_{0}^{\infty} E\left[\left|r_{n}\right|^{l} \mid g_{n}, s_{n}\right] f_{\left|g_{n}\right|}\left(\left|g_{n}\right|\right) d\left|g_{n}\right| \\
& =2^{\frac{l}{2}} \sigma_{w}^{l} \frac{\Gamma\left(\frac{l}{2}+1\right)}{\Gamma(m)}\left(\frac{m}{\alpha_{g}^{2}}\right)^{m} \\
& \int_{0}^{\infty} e^{-\left(\frac{m}{\alpha_{g}^{2}}+\frac{\left|s_{n}\right|^{2}}{2 \sigma_{w}^{2}}\right)\left|g_{n}\right|^{2}}\left(\left|g_{n}\right|^{2}\right)^{m-1} \\
& \times{ }_{1} F_{1}\left(\frac{l}{2}+1 ; 1 ; \frac{\left|s_{n}\right|^{2}}{2 \sigma_{w}^{2}} \times\left|g_{n}\right|^{2}\right) d\left|g_{n}\right|^{2} .
\end{aligned}
$$

From [36, section 7.621],

$$
\int_{0}^{\infty} e^{-s t} t^{b-1}{ }_{1} F_{1}(a ; c ; k t) d t=\Gamma(b) s_{2}^{-b} F_{1}\left(a, b ; c ; k s^{-1}\right)
$$

and $\rho=\frac{\alpha_{g}^{2}}{2 \sigma_{w}^{2}}$.

Hence,

$$
\begin{aligned}
E\left[\left|r_{n}\right|^{l} \mid s_{n}\right]=2^{\frac{l}{2}} & \Gamma\left(\frac{l}{2}+1\right) \sigma_{w}^{l}\left(\frac{m}{m+\rho\left|s_{n}\right|^{2}}\right)^{m} \\
& \times{ }_{2} F_{1}\left(\frac{l}{2}+1, m, 1 ; \frac{\rho\left|s_{n}\right|^{2}}{m+\rho\left|s_{n}\right|^{2}}\right),
\end{aligned}
$$

which leads to (7) after removing the condition on $s_{n}$.

According to [34], ${ }_{2} F_{1}(a, b, c ; y)=(1-y)^{-b}{ }_{2} F_{1}$ $\left(c-a, b, c ; \frac{y}{y-1}\right)$, so we can rewrite (7) in the form

$$
\begin{aligned}
E\left[\left|r_{n}\right|^{l}\right]=2^{\frac{l}{2}} \Gamma\left(\frac{l}{2}+1\right) & \sigma_{w}^{l} \int_{0}^{\infty} e^{-\left|s_{n}\right|^{2}} \\
& \times{ }_{2} F_{1}\left(\frac{-l}{2}, m, 1 ; \frac{-\rho\left|s_{n}\right|^{2}}{m}\right) d\left|s_{n}\right|^{2} .
\end{aligned}
$$

Defining $u=\frac{\rho}{m}\left|s_{n}\right|^{2}$, we end up with

$$
\begin{aligned}
M_{2 l} \triangleq E\left[\left|r_{n}\right|^{2 l}\right]=2^{l} & \Gamma(l+1) \sigma_{w}^{2 l} \frac{m}{\rho} \\
& \times \int_{0}^{\infty} e^{-\frac{m}{\rho} u}{ }_{2} F_{1}(-l, m, 1 ;-u) d u
\end{aligned}
$$

From [36], we know that

$\int_{0}^{\infty} e^{-\lambda x} x_{2}^{\gamma-1} F_{1}(\alpha, \beta, \delta ;-x) d x=\frac{\Gamma(\delta) \lambda^{-\gamma}}{\Gamma(\alpha) \Gamma(\beta)} E(\alpha, \beta, \gamma ; \delta ; \lambda)$, where $E($.$) denotes the MacRobert's E-function. Based on$ the relation between the MacRobert's E-function and the 
generalized hypergeometric function, we can write

$$
E\left(\begin{array}{c}
a_{p} \\
b_{q}
\end{array} \mid x\right)=\frac{\prod_{j=1}^{p} \Gamma\left(a_{j}\right)}{\prod_{j=1}^{q} \Gamma\left(b_{j}\right)} p F_{q}\left(\begin{array}{c}
a_{p} \\
b_{q}
\end{array} \mid-\frac{1}{x}\right) .
$$

We can write (34) as

$$
M_{2 l}=2^{l} \Gamma(l+1) \sigma_{w}^{2 l} \times{ }_{3} F_{1}\left(-l, m, 1 ; 1 ;-\frac{\rho}{m}\right) \quad \forall m \in \mathbb{R}
$$

Based on the standard definition of the generalized hypergeometric function

$$
{ }_{p} F_{q}\left(\begin{array}{c}
a_{p} \\
b_{q}
\end{array} \mid x\right)=\sum_{n=0}^{\infty} \frac{\prod_{j=1}^{p} a_{j}^{(n)}}{\prod_{j=1}^{q} b_{j}^{(n)}} \frac{x^{n}}{n !},
$$

(36) can be rewritten as

$$
M_{2 l}=2^{l} \Gamma(l+1) \sigma_{w}^{2 l} \sum_{j=0}^{\infty} \frac{m^{(j)}(-l)^{(j)}}{j !} \times\left(-\frac{\rho}{m}\right)^{j} . \quad \forall m \in \mathbb{R}
$$

Knowing that $(-l)^{(j)}=(-1)^{j} l_{(j)}, \frac{l_{(j)}}{j !}=\left(\begin{array}{l}l \\ j\end{array}\right)$, and $l>j$, we can reach the result of Theorem 1 ,

$$
M_{2 l}=2^{l} \Gamma(l+1) \sigma_{w}^{l}\left(1+\sum_{j=1}^{l}\left(\begin{array}{l}
l \\
j
\end{array}\right) \frac{m^{(j)}}{m^{j}} \rho^{j}\right) .
$$

\section{APPENDIX B}

\section{OFDM SIGNALS AS GAUSSIAN-DistribUtED SignALS}

An OFDM signal, $s_{n}$ can be written as

$$
s_{n}=\frac{1}{\sqrt{N}} \sum_{k=0}^{N-1} x_{k} e^{j 2 \pi \frac{k n}{N}}
$$

where $x_{k}=I_{k}+j Q_{k}, I_{k}$ and $Q_{k}$ are in-phase and quadrature components respectively, $k \in\{0,1, \ldots, N-1\}$ is the subcarrier index and $x_{k}$ is the baseband symbol, which is taken from any arbitrary constellation. Assuming that $x_{k}$ has zero-mean and the constellation power is normalized, according to the central limit theorem, and for a large number of sub-carriers $N$, it can be assumed that $s_{n}$ is a complex Gaussian random variable with zero-mean and normalized power.

\section{ACKNOWLEDGMENT}

The authors would like to thank the anonymous reviewers and editor for their constructive comments that helped improve the quality of this paper and for the suggestions to improve the proof of Theorem 1 .

\section{REFERENCES}

[1] T. Yucek and H. Arslan, "A survey of spectrum sensing algorithms for cognitive radio applications," IEEE Commun. Surveys Tuts., vol. 11, no. 1, pp. 116-130, 2009.

[2] A. Ghasemi and E. S. Sousa, "Spectrum sensing in cognitive radio networks: Requirements, challenges and design trade-offs," IEEE Commun. Mag., vol. 46, no. 4, pp. 32-39, Apr. 2008.
[3] H. Al-Hmood, R. S. Abbas, A. Masrub, and H. S. Al-Raweshidy, "An estimation of primary user's SNR for spectrum sensing in cognitive radios," in Proc. 3rd INTECH, London, U.K., Aug. 29-31, 2013, pp. 479-484.

[4] M. Fujii and Y. Watanabe, "A study on SNR estimation for cognitive radio," in Proc. IEEE ICUWB, Syracuse, NY, USA, Sep. 17-20, 2012, pp. $11-15$.

[5] S. K. Sharma, S. Chatzinotas, and S. Ottersten, "SNR estimation for multi-dimensional cognitive receiver under correlated channel/noise," IEEE Trans. Wireless Commun., vol. 12, no. 12, pp. 6392-6405, Dec. 2013.

[6] K. Seshukumar, R. Saravanan, and M. S. Suraj, "Spectrum sensing review in cognitive radio," in Proc. ICEVENT, Tiruvannamalai, India, Jan. 7-9, 2013, pp. 1-4.

[7] D. R. Pauluzzi and N. C. Beaulieu, "A comparison of SNR estimation techniques for the AWGN channel," IEEE Trans. Commun., vol. 48, no. 10, pp. 1681-1691, Oct. 2000.

[8] F. Bellili, A. Stéphenne, and S. Affes, "Cramer-Rao lower bounds for NDA SNR estimates of square QAM modulated transmissions," IEEE Trans. Commun., vol. 58, no. 11, pp. 3211-3218, Nov. 2010.

[9] F. Bellili, A. Stéphenne, and S. Affes, "SNR estimation of QAMmodulated transmissions over time-varying SIMO channels," in Proc. IEEE ISWCS, Reykjavik, Iceland, Oct. 21-24, 2008, pp. 199-203.

[10] A. Das, "NDA SNR estimation: CRLBs and EM based estimators," in Proc. IEEE TENCON, Hyderabad, India, Nov. 18-21, 2008, pp. 1-6.

[11] J. Descure, F. Bellili, and S. Affes, "ML estimator based on the EM algorithm for subcarrier SNR estimation in multicarrier transmissions," in Proc. AFRICON, Nairobi, Kenya, Sep. 23-25, 2009, pp. 1-5.

[12] M. A. Boujelben, F. Bellili, S. Affes, and A. Stéphenne, "EM algorithm for non-data-aided SNR estimation of linearly-modulated signals over SIMO channels," in Proc. IEEE GLOBECOM, Honolulu, HI, USA, Nov. 30Dec. 4, 2009, pp. 1-6.

[13] T. A. Summers and S. G. Wilson, "SNR mismatch and online estimation in Turbo decoding," IEEE Trans. Commun., vol. 46, no. 4, pp. 421-423, Apr. 1998.

[14] H. Xu, Z. Li, and H. Zheng, "A non-data-aided SNR estimation algorithm for QAM signals," in Proc. ICCCAS, Chengdu, China, Nov. 30-Dec. 4, 2009, vol. 2, pp. 999-1003.

[15] O. H. Tekbas, "Blind SNR estimation for limited time series," Chaos Solitons Fractals, vol. 33, no. 5, pp. 1497-1504, Aug. 2007.

[16] R. Matzner, "An SNR estimation algorithm for complex baseband signals using higher order statistics," Facta Universitatis (Nis), vol. 6, no. 1, pp. 41-52, 1993.

[17] A. Wiesel, J. Goldberg, and H. Messer, "Non-data-aided signal-to-noiseratio estimation," in Proc. IEEE ICC, New York, NY, USA, Apr. 28May 1, 2002, vol. 1, pp. 197-201.

[18] A. Wiesel, J. Goldberg, and H. Messer, "SNR estimation in time-varying fading channels," IEEE Trans. Commun., vol. 54, no. 5, pp. 841-848, May 2006.

[19] R. Lopez-Valcarce and C. Mosquera, "Sixth-order statistics-based non-data-aided SNR estimation," IEEE Commun. Lett., vol. 11, no. 4, pp. 351-353, Apr. 2007.

[20] R. Lopez-Valcarce, C. Mosquera, and W. Gappmair, "Iterative envelopebased SNR estimation for non-constant modulus constellations," in Proc. IEEE 8th Workshop SPAWC, Helsinki, Finland, Jun. 17-10, 2007, pp. 1-5.

[21] M. Alvarez-Diaz, R. Lopez-Valcarce, and C. Mosquera, "SNR estimation for multilevel constellations using higher-order moments," IEEE Trans. Signal Process., vol. 58, no. 3, pp. 1515-1526, Mar. 2010.

[22] A. Stéphenne, F. Bellili, and S. Affes, "Moment-based SNR estimation for SIMO wireless communication systems using arbitrary QAM," in Proc. 41st Asilomar Conf. Signals, Syst. Comput., Pacific Grove, CA, USA, Nov. 4-7, 2007, pp. 601-605.

[23] A. Stéphenne, F. Bellili, and S. Affes, "Moment-based SNR estimation over linearly-modulated wireless SIMO channels," IEEE Trans. Wireless Commun., vol. 9, no. 2, pp. 714-722, Feb. 2010.

[24] M. A. Boujelben, F. Bellili, S. Affes, and A. Stéphenne, "SNR estimation over SIMO channels from linearly modulated signals," IEEE Trans. Signal Process., vol. 58, no. 12, pp. 6017-6028, Dec. 2010.

[25] F. Socheleau, A. Ayssa-El-Bey, and S. Houcke, "Non data-aided SNR estimation of OFDM signals," IEEE Commun. Lett., vol. 12, no. 11, pp. 813-815, Nov. 2008.

[26] S. A. Kim, D. G. An, H. Ryu, and J. Kim, "Efficient SNR estimation in OFDM system," in Proc. IEEE RWS, Phoenix, AZ, USA, Jan. 16-19, 2011, pp. 182-185.

[27] A. Ramesh, A.Chockalingam, and L. B. Milstein, "SNR estimation in generalized fading channels and its application to turbo decoding," in Proc. IEEE ICC, Helsinki, Finland, Jun. 11-14, 2001, vol. 4, pp. 1094-1098. 
[28] A. Ramesh, A. Chockalingam, and L. B. Milstein, "SNR estimation in Nakagami-m fading with diversity combining and its application to Turbo decoding," IEEE Trans. Commun., vol. 50, no. 11, pp. 1719-1724, Nov. 2002.

[29] S. A. Dianat, "SNR estimation in Nakagami fading channels with arbitrary constellation," in Proc. IEEE ICASSP, Honolulu, HI, USA, Apr. 15-20, 2007, vol. 2, pp. 325-328.

[30] Y. Yeh and S. Chen, "An efficient fast-fading channel estimation and equalization method with self ICI cancellation," in Proc. EUSIPCO, Vienna, Austria, Sep. 6-10, 2004, pp. 449-452.

[31] R. K. Mallik, "A new statistical model of the complex Nakagami-m fading gain," IEEE Trans. Commun., vol. 58, no. 9, pp. 2611-2620, Sep. 2010.

[32] S. M. Kay, Fundamentals of Statistical Signal Processing, Volume I: Estimation Theory. Englewood Cliffs, NJ, USA: Prentice-Hall, 1998.

[33] J. G. Proakis, Digital Communications, 4th ed. New York, NY, USA: McGraw-Hill, 2001.

[34] F. Beukers, "Arithmetic and geometry around hypergeometric functions," Progress Math., vol. 260, pp. 23-42, 2007.

[35] A. Abdi and M. Kaveh, "Performance comparison of three different estimators for the nakagami $\mathrm{m}$ parameter using Monte Carlo simulation," IEEE Commun. Lett., vol. 4, no. 4, pp. 119-121, Apr. 2000.

[36] I. S. Gradshteyn and I. M. Ryzhik, Table of Integrals, Series, Products, 7th ed. New York, NY, USA: Academic, 2007.

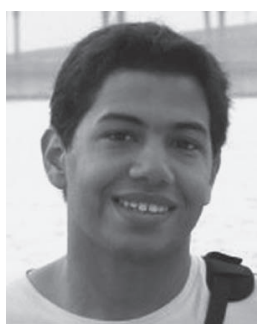

Mohammed Hafez (S'07) received the B.Sc. degree in electrical engineering from Alexandria University, Alexandria, Egypt, in 2010, and the M.Sc. degree in electrical engineering from the same university, in 2014. He is a graduate assistant in the wireless communications and signal Processing group, University of South Florida, Tampa, FL, and is currently working towards the Ph.D. degree in electrical engineering from the same university. He was a research assistant in the department of electrical engineering at Qatar University, Doha, Qatar, from 2011 to 2014. He was also a research assistant in the department of electrical engineering at Alexandria University, Alexandria, Egypt, from 2010 to 2011.

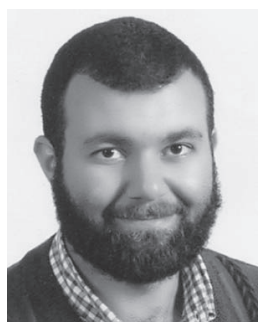

Tamer Khattab (M'94) received the B.Sc. and the M.Sc. degrees in electronics and communications engineering from Cairo University, Giza, Egypt, and received the $\mathrm{Ph} . \mathrm{D}$. degree in electrical and computer engineering from the University of British Columbia (UBC), Vancouver, BC, Canada, in 2007. He has been an assistant professor of Electrical Engineering at Qatar University (QU) since 2007. He is also a senior member of the technical staff at Qatar Mobility Innovation Center (QMIC), an R\&D center owned by QU and funded by Qatar Science and Technology Park (QSTP). Between 2006 and 2007 he was a postdoctoral fellow at the University of British Columbia working on prototyping advanced Gigabit/sec wireless LAN baseband transceivers. During 2000-2003 Dr. Khattab joined Alcatel Canada's Network and Service Management R\&D in Vancouver, BC, Canada as a member of the technical staff working on development of core components for Alcatel 5620 network and service manager. Between 1994 and 1999 he was with IBM wtc. Egypt as a software development team lead working on development of several client-server corporate tools for IBM labs. Dr. Khattab's research interests cover physical layer transmission techniques in optical and wireless networks, information theoretic aspects of communication systems and MAC layer protocol design and analysis.

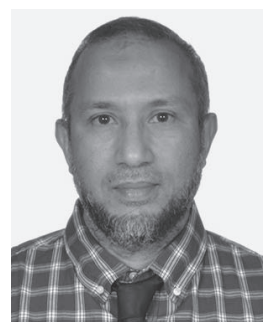

Hossam M. H. Shalaby (S'83-M'91-SM'99) was born in Giza, Egypt, in 1961. He received the B.S. and M.S. degrees from Alexandria University, Alexandria, Egypt, in 1983 and 1986, respectively, and the Ph.D. degree from the University of Maryland at College Park in 1991, all in electrical engineering.

In 1991, he joined the Electrical Engineering Department, Alexandria University, and was promoted to Professor in 2001. Currently he is on leave from Alexandria University, where he is the chair of the Department of Electronics and Communications Engineering, School of Electronics, Communications, and Computer Engineering, Egypt-Japan University of Science and Technology (E-JUST), New Borg EL-Arab City, Alexandria, Egypt. From December 2000 to 2004, he was an Adjunct Professor with the Faculty of Sciences and Engineering, Department of Electrical and Information Engineering, Laval University, Quebec, QC, Canada. From September 1996 to February 2001, he was on leave from the Alexandria University. From September 1996 to January 1998, he was with the Electrical and Computer Engineering Department, International Islamic University Malaysia, and from February 1998 to February 2001, he was with the School of Electrical and Electronic Engineering, Nanyang Technological University, Singapore. His research interests include optical communications, optical CDMA, optical burst-switching, OFDM technology, and information theory.

Prof. Shalaby has served as a student branch counselor at Alexandria University, IEEE Alexandria and North Delta Subsection, from 2002 to 2006, and served as a chairman of the student activities committee of IEEE Alexandria Subsection from 1995 to 1996 . He received an SRC fellowship from 1987 to 1991 from Systems Research Center, Maryland; State Excellence Award in Engineering Sciences in 2007 from Academy of Scientific Research and Technology, Egypt; Shoman Prize for Young Arab Researchers in 2002 from Abdul Hameed Shoman Foundation, Amman, Jordan; State Incentive Award in Engineering Sciences in 1995 and 2001 from Academy of Scientific Research and Technology, Egypt; University Excellence Award in 2009 from Alexandria University; and University Incentive Award in 1996 from Alexandria University. He is a member of the IEEE Photonics Society and The Optical Society (OSA). 